\title{
Accuracy of ECG in assessment of postmyocardial infarction left ventricular function \& its comparison with echocardiography
}

\author{
Dr Prashant Gowardhan ${ }^{1}$, Dr R. Sharma ${ }^{2}$ \\ ${ }^{1}$ (Consultant physician, Rani Durgavati University, India) \\ ${ }^{2}$ (Medicine department, N.S.C.B. Medical college, Rani Durgavati University, India)
}

\section{Introduction}

Coronary heart disease is the leading cause of mortality and morbidity in the developed countries and is fast catching up with nutritional and infectious diseases in the developing countries also. Ischemic heart disease has been branded as "Modern Edpidemic" by the WHO. ${ }^{[1]}$

The diagnosis of an acute myocardial infarction (MI) is typically based upon the history, electrocardiogram, and cardiac enzymes, particularly serum troponins and creatine kinase MB fraction (CKMB). Although not routinely performed for diagnosis, echocardiography is an accurate, noninvasive test that is able to detect evidence of myocardial ischemia or necrosis.

The major determinate of the immediate and long-term outcome of acute myocardial infarction are size of the myocardial infarct and functional status of the residual myocardium. ${ }^{[2]}$ This requires CPK-MB estimation and quantitation, thallium perfusion scanning, technetium perfusion scanning, antimyosin antibody scanning, radionuclide angiocardiography, position emission tomography (PET) coronary angiography and ventriculography exercise stress testing, holter monitoring and echocardiogram. ${ }^{[3]}$

However, most of these investigation are very expensive, consume time and resources and above all, not available in many centers especially in a developing country like India. Hence they are of limited value in mass population usage.

The value of electrocardiogram (ECG) in the diagnosis and localization of a myocardial infarct is well established ${ }^{[4]}$ However, its usage in measuring the size of the infarct and assessing left ventricular function is a relatively new concept.

The two dimensional echocardiography made it possible to detect wall motion abnormalities which are used to differentiate reliably between patient with myocardial ischaemia or early infarction and patient with nonischaemic chest pain. ${ }^{[5]}$ The major advantages of the two dimensional echocardiography is its non-invasive use and repeatability of the examination. ${ }^{[6]}$ The wall motion abnormalities detected using the echocardiography can be used safely in the diagnosis of myocardial infarction in the appropriate setting when electrocardiogram is non diagnostic. This technique has shown good correlation with functional infarct size with studies using thallium ventriculography, coronary angiography, peak creatinine phosphokinase levels and nuclear ventriculography. ${ }^{[3]}$ 2D-Echocardiography, because of its ability to determine global and regional ventricular function is suited ideally for determining early and late prognosis in patients with acute myocardial infarction. ${ }^{[5]}$ When performed early in the course of myocardial infarction 2D-echocardiography can help stratify high and low risk groups. Now a days measurement of left ventricular function is made by means of 'Ejection Fraction'. Ejection fraction is global index of extent of ventricular fiber shortening and provides useful measure of overall left ventricular pump function.

ECG and 2-D echocardiography both are feasible and acceptable investigation to diagnose LV dysfunction. The outcome may be improved when the results of the two are compared for final evaluation.

\section{Material And Methods}

The present study was carried out in fifty cases of acute myocardial infarction patients in the tertiary care Hospital .

All the patients who were admitted in the Medical wards who had demonstrated Q waves in their electrocardiogram were included in this study and screened.This also included patients shifted from coronary care units convalescing from acute infarct.

Patients who had a history of DIABETES MELLITUS HYPERTENSION, CHRONIC RENAL FAILURE and/or on treatment for the same were excluded from the study as these diseases may affect the myocardium directly. Patients with non- Q wave infarcts, ${ }^{[7]}$ Patient with conduction blocks assessed by electrocardiogram and Patients who showed electrocardiography evidence of Right or Left Ventricular hypertrophy by ESTES criteria were excluded. ${ }^{[8]}$ Also,patients who had echocardiography evidence of valvular heart diseases of any of the four valves were excluded. 
Standard 12 lead surface electocardiography was performed by using single channel 108 T/MK (BPL) Machine on the day of the echocardiogram. The electrodes were placed in the standard positionss. ${ }^{[9]}$

The Tenth Bethesda conference (1978)102 classified infarcts on the basis of presence of Q waves. The electrocardiogram thus obtained was analysed by using the Modified Selvester Scoring System. ${ }^{[10]}$ After carefully scrutinizing each lead for duration and amplitude of Q.R and S wave score from each of 10 leads considered were added and expressed as a total score out of possible score of 29.Angiocardiographic formula Left Ventricular Ejection Fraction $=60-\quad(3 \mathrm{x}$ QRS score $)$ was applied and projected ejection fraction was calculated and compared with the predicted ejection fraction was calculated and compared with the predicted ejection fraction by echo.

Once an electrocardiographic recording was made, the score and the expected ejection fraction calculated from the score were noted. Echo was done (both 2-D \& M-mode). The subjects were examined in 30 degree and 60 degree left lateral decubitus position during quiet respiration. This particular position utilizes gravity bringing heart more close to the sternum and facilitating the recording of cardiac echoes.

The left ventricular volume was derived from "bullet formula". Volume $=5 / 6 \times \mathrm{A} \times \mathrm{L}$

$\mathrm{A}=$ Area of Left ventricular cavity at papillary muscle Level.

$\mathrm{L}=$ Length of left ventricular cavity from apex to mitral annulus.

Ejection fraction is calculated by using formula -

End diastolic volume - End systolic volume

Ejection Fraction = ----------on 100

End diastolic volume

which is expressed as percentage.

By using a M-mode view at the level of mitral valve, motions are recorded. The distance between peak excursion of anterior leaflet of mitral valve (E-point) \& Interventricular septum is measured \& expressed as EPSS (E point septal separation)

Once the echocardiography measurement are made, they were compared with the ECG score and the ECG assessment of left ventricular fraction.

\section{Observations}

Total 50 patients were included in the study.

The largest group was formed by patients in age group of 51-60 (46\%). The overall Mean age was 54.7 years. Mean age in cases of male patients 54.3 years while in case of females mean age was 57.6 years.

The overall mean ECG score was $5.5(\mathrm{n}=50)$ while in patients with left ventricular failure mean ECG score was (7.6) found to be significant higher.

$54 \%$ of the patients studied were in acute convalescing stage and two third of them belonged to inferior infarct group. The maximum number of cases had duration of infract ranging from 2 weeks to 1 year.

Though coefficient of correlation is positive but it shows a week correlation between ECG score and EPSS among the non LV failure cases. Statistically it is insignificant $(\mathrm{t}=1.67)$.

The ejection fraction estimated by ECG correlated well with echocardiograph assessment and the coefficient of correlation was $r=0.82$ which was found to be statistically highly significant $(t=9.91, p<0.001)$

The association between the predicted left ventricular ejection fraction by ECG and echocardiography was stronger when the ejection fraction was low.

Patients with anterior wall infarct have large area of infarct and higher incidence of left ventricular failure.

Patients with combined infarct have more extensive area of involvement of muscle mass more chances of left ventricular pump failure

\section{Discussion}

The role of QRS complex in predicting the volume of necrotic myocardium has become clear by series of studies. The invention of QRS scoring system by Selvester at el $(1969)^{[11]}$ and its modification by Wagner revealed the role of QRS scoring system in estimating the size of myocardial infarction. Robin et al $(1983)^{[12]}$ demonstrated that QRS score relates well with the extent of myocardial damage as assessed by peak creatine kinase levels. Since the volume of the necrotic myocardium in infarction is one of the best indicators of left ventricular function as suggested by many reports and that QRS score correlates well with the extent of myocardial necrotic volume after infarction, the present study was taken up with the prospect in mind to study the left ventricular function after acute myocardial infarction with QRS scoring system and its correlation with echocardiography.

In the present study of 50 cases, patients were evenly distributed in the anterior infarct group (44\%) and inferior infarct group (48\%) (Table No. 1). Eighty Six percent of subjects were male which confirms the universal trend of study from all over the world that show a male preponderance in sex distribution of coronary artery disease. 
Maximum number of patients belong to the age group of 51-60 followed by 40-50. The sex distribution is also similarly high in this age group. This correlates well with known Indian datas. ${ }^{[1]}$ Banerjee et al (1960) suggested that coronary artery disease is mainly a disease of middle and old age. The cases of anterior infarct group were comparatively younger to other groups of M1 and the mean age was found to be 49.59 years.

A trend was observed that in $38.63 \%$ cases with LV failure, infarct duration was less than 1 year while the $12 \%$ of LV failure cases had infract duration of more than 1 year. Though this differences was statistically insignificant but the $95 \%$ confidence probability of LV failure with increasing age of infarct (table 3 ). It is commonly seen that the complications of acute infarct like post infarct failure are common in first year of infarction.

The anterior infarction subgroup had a new $\mathrm{Q}$ waves $\geq 30$ milli seconds that were localized to one or more of precordial leads. The inferior infarct sub groups revealed new $\mathrm{Q}$ waves $\geq 30$ milli seconds in lead AVF. Horan et al (1971) ${ }^{[13]}$ fund that the Q waves of more than thirty millisecond duration were falsely positive in $4 \%$ of patients.

The previous studies by Idekar et al ${ }^{[14]}$ and Roark et at ${ }^{[15]}$ (1983), showed that modified Selvester QRS score of ECG correlates with infarct size, and a score of 3 or above is highly significant.

Since the present study included patients who had definitive evidence of infarction, the overall mean ECG score was 5.5. The ECG score was higher for the anterior wall infarction group than the inferior wall infarction group (Table No. 4). This indicated that the anterior infarcts were larger in size than the inferior infarcts, which could explain the tendency of the anterior infarcts to undergo pump failure.

The ECG score for inferior wall infarction was lower than that of anterior wall. However, the echo ejection fraction was higher than the ECG estimates.

When the QRS scoring system was used to determine the left ventricular ejection fraction, the estimated ejection fraction correlated well with the echocardiographic ejection fraction to the point of statistical significance .

Askenazi et al (1978) ${ }^{[16]}$ assessed LVEF by QRS complex from sum of R wave amplitude from six precordial leads and correlated with LVEF by echo.

Berdia et al (1991) ${ }^{[17]}$ observed that the mean LVEF calculated by ECG scoring and the mean LVEF predicted by echocardiography were quite comparable but were statistically insignificant.

Samuel et al (1992) ${ }^{[18]}$ found a positive correlation between LVEF estimated by QRS scoring system and the LVEF estimated by echocardiography with a correlation coefficient of $r=0.78$ the point of statistical significance.

Thus, the modified Selvester scoring system is capable of predicting left ventricular function with good correlation. The formula used in this study is the angiographic formula for ejection fraction $=60-(3 \mathrm{x}$ QRS score).

The same scoring system was able to show good correlation especially when the left ventricular ejection fraction was lower than when they were not affected. This is significant because, it is the patient with decreased left ventricular function, who requires intervention.

Patients with evidence of LV failure had significantly raised ECG score, diminished ejection fraction, elevated EPSS and multiple contraction abnormalities. The LV failure was more common in anterior and global infarct than inferior infarcts due to more extensive involvement of large area of muscle mass. This is further corroborated by the fact that all 4 cases that came with combined infarction had LVF $(100 \%)$. Though this study had larger number of patient from acute convalescing infarct group, LV failure was commonest in two weeks to one-year post infarct group. An ECG score of seven or more was present in patient with LV failure EPSS was also significantly increased (almost doubled) in patient with LV failure with mean EPSS (16.3) (normal 8mm) hence EPSS correlated directly with ECG score to the point of statistical significance in LV failure patients. This also correlates with the previously established fact that elevated EPSS is an indicator of heart failure but people with heart failure can have normal EPSS because the patients may not have dilated heart. Also it is a more specific maker of coronary heart disease.

In the present study the QRS scoring system proved to be an accurate predictor of LV function and that the ECG can provide indirect and quantitative measure of infarct size and LV function in non invasive manner in day to day life.

\section{Conclusion}

ECG scoring system provides a reliable indirect estimate of infarct size, regional and global left ventricular function comparable to echocardiography.It can be used to separate patients with left ventricular failure and those without left ventricular failure.

E point septal separation showed a good correlation with ECG score in patients with left ventricular failure to the point of statistical significance. So this method of left ventricular function assessment can be used routinely for screening large population and for bedside management of individual patient. It was found to be 
cost effective, easily available, repeatable and easily applicable with sufficient accuracy in assessing left ventricular function in patients with acute myocardial infarction. Therefore we can prognosticate the patients of myocardial infarction by

simple non invasive method of ECG scoring as overall prognosis depends

on post myocardial infarct left ventricular function.

\section{References}

[1]. Park J. E. - Text book of Preventive and social medicine $16^{\text {th }}$ edition 2000.

[2]. Geltman, E.M. Ehsani A.A., et al., the influenced of location and extent of infraction of location and extent of infraction on longterm ventricular dysarrhyimia and mortality. Circulation 1979:60:271-279.

[3]. Echocardiography - feigenbaum $5^{\text {th }}$ Edition -1994 zea and Febiger

[4]. Horan L. G. flowers N.C. et al Significance of diagnostic Q waves in myocardial infarctions. Circulation 43;428:1971.

[5]. Ortzega E. Charuzi. Y Honma H. 2-D in acute coronary care. In Schapira JN, Harold JG, 2-D echo \& cardiac Doppler. $2^{\text {nd }}$ edition Balti more Williams, B. Wilkins 1990.

[6]. Widimsky P, Cervenka, Gregor P, et al, A longitudinal echocardiogram study. European Heart Journal (1985)(6) 759-965.

[7]. Ogewa J. Hiramoro, K. et al., Classification of non Q wave myocardial infarction according to electro cardiographic changes $\mathrm{Br}$. Heart Journal 54;473-1985.

[8]. Lekven J. Chattergee J. et al., Influence of left ventricular dimensions on endocardial and epicardial QRS amplitude and St, Segment elevations during acute myocardial ischemia Circulations 61;679-1980.

[9]. An Introduction to Electrocardiography - Leo Schamroth Seventh Edition - 1990.

[10]. Wagner G.D. Freye C.J. et al. Evaluation of a QRS Scoring for estimating myocardial infarct size. Circulation 65:342-1982.

[11]. Selvester, R. s. Soloman J. C. \& Gillespie TL, Digital computer model of total body electrocardiography surface map circulation 38:384-1968.

[12]. Robin GS, Shen WF, Kelly DT, Harris PJ : QRS scoring system for estimating myocardial infarct size. Clinical, angiographic and prognostic correlation. J. Am. Coll. Cardiol. Vol. 2, 148-144, 1983.

[13]. Horan L. G. flowers N.C. et al Significance of diagnostic Q waves in myocardial infarctions. Circulation 43;428:1971.

[14]. Idekar, R.E. Wagner G.S. et al., Evaluation of a QRS Scoring system for estimating myocardial infarct size. II. Correlation with quantitative anatomic findings for anterior infarcts. Am. JI. Cardiology 49:1604-1982.

[15]. Gitler B. Cooper J. A., Letter to editor. A QRS scoring system for assessment of LVEFafter AMI. New Eng. J. Med. 306 No. 23:1423:1982.

[16]. Askenazi J. Parasi A.F. Braunwald E. et al. Value of the QRS complex in assessing left ventricular ejection fraction. Am. J. Cardial 41;494:1978.

[17]. Berdai S. et al (1991) : Study of QRS scoring system and it correlation with echo in cases of AMI. (1991).

[18]. Samuel D. et al 1992 : Assessment of LV function in post MI from ECG (1992).

Table 1: Sex distribution in different groups of MI Patients

\begin{tabular}{|l|l|l|l|l|l|}
\hline S. No. & Type of Infarct (Group) & Male & Female & Total & Percentage \\
\hline 1. & Anterior (A) & 19 & 3 & 22 & $44 \%$ \\
\hline 2. & Inferior (B) & 20 & 4 & 24 & $48 \%$ \\
\hline 3. & Combined (C) & 4 & - & 4 & $8 \%$ \\
\hline Total & & $\mathbf{4 3}$ & $\mathbf{7}$ & $\mathbf{5 0}$ & $\mathbf{1 0 0} \%$ \\
\hline
\end{tabular}

Table 2:Profile of patient with left ventricular Failure

\begin{tabular}{|l|l|l|l|}
\hline S. No. & Type of Infarct & No. of patients & Percentage \\
\hline 1. & Anterior & 13 & 62 \\
\hline 2. & Inferior & 4 & 19 \\
\hline 3. & Combined & 4 & 19 \\
\hline Total & & $\mathbf{2 1}$ & $\mathbf{1 0 0}$ \\
\hline
\end{tabular}

Left ventricular failure was seen in $42 \%$ of total cases and largest subgroup was formed by patients with anterior wall M1 (62\%) followed by patients with inferior wall in M1 (19\%).

Table 3: Duration of infarct with and without left ventricular Failure

\begin{tabular}{|l|l|l|l|l|}
\hline & With LVF & Without LVF & Total & Percentage \\
\hline$<1$ yrs & $17(38.63 \%)$ & 27 & 44 & 88 \\
\hline$>1$ yrs & $4(66.67 \%)$ & 2 & 6 & 12 \\
\hline Overall & 21 & 29 & 50 & 100 \\
\hline
\end{tabular}

$88 \%$ cases had infarct duration of less than 1 year out of which $38.63 \%$ had LVF where as $12 \%$ cases had infarct duration of more than 1 year out of which $66.67 \%$ had LVF. 
Accuracy of ECG in assessment of postmyocardial infarction left ventricular function \& its

Table 4: Mean ECG score in different groups of MI patients with \& without LV failure

\begin{tabular}{|c|c|c|c|c|c|}
\hline S. No. & Type of Infaret & 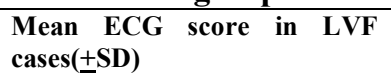 & $\begin{array}{l}\text { Mean } \\
\text { LVF ca }\end{array}$ & $\begin{array}{l}\text { score } \\
\text { D) }\end{array}$ & $Z$ value \\
\hline 1. & Anterior & $\begin{array}{l}7.46 \\
( \pm 1.76) \mathrm{n}=13\end{array}$ & $\begin{array}{l}3.55 \\
( \pm 1.74) \\
\end{array}$ & $\mathrm{n}=9$ & $\begin{array}{l}\mathrm{Z}=5.16 \\
\mathrm{P}<0.001\end{array}$ \\
\hline 2. & Inferior & $6.25( \pm 0.96)$ & $\begin{array}{l}3.45 \\
(+1.09)\end{array}$ & $\mathrm{n}=20$ & $\begin{array}{l}\mathrm{Z}=5.20 \\
\mathrm{P}<0.001\end{array}$ \\
\hline 3. & combined & $\begin{array}{ll}10.5 & ( \pm 1.29) \\
\mathrm{n}=4 & \end{array}$ & - & & - \\
\hline 4. & Overall & $\begin{array}{ll}7.66 & ( \pm 2.22) \\
\mathrm{n}=21\end{array}$ & $\begin{array}{l}3.58 \\
(+1.45)\end{array}$ & $n=29$ & \\
\hline
\end{tabular}

